\title{
High-Selectivity Reflectionless Unbalanced-to-Balanced Filtering Power Combiner
}

\author{
Zihui ZHU, Zhongbao WANG, Yu BAI, Hongmei LIU, Shaojun FANG \\ School of Information Science and Technology, Dalian Maritime University, Dalian, Liaoning 116026, China \\ zhu_zihui@dlmu.edu.cn,wangzb@dlmu.edu.cn \\ Submitted February 4, 2021 / Accepted June 2, 2021
}

\begin{abstract}
A high-selectivity reflectionless unbalanced-tobalanced (UTB) filtering power combiner is proposed. The proposed power combiner composes of two absorbing branches, two filtering sections, two transmission lines, a grounded resistor, and a phase inverter. Two transmission zeros respectively located at the lower and upper sides of the passband are achieved by the filtering sections, resulting in high selectivity. The input-port reflectionless response in the bandstop region is obtained by the absorbing branch. A 1.0-GHz UTB filtering power combiner is designed and fabricated. Finally, the measurement performances are given in this paper. The input-reflection absorptive bandwidth and transmission bandwidth are measured as $545 \mathrm{MHz}$ and $132 \mathrm{MHz}$, respectively. The absorptive bandwidth is 4.12 times the transmission bandwidth.
\end{abstract}

\section{Keywords}

Power combiner, reflectionless response, unbalancedto-balanced, high-selectivity filtering

\section{Introduction}

Power combiner is capable of summing signal power, which has been widely used in balanced amplifier [1] and the linear amplification system using nonlinear components [2]. Furthermore, power amplifiers with high output power have become more and more important in $5 \mathrm{G}$ mobile communication and radar systems. As the operating frequency increases, the power handling capability of semiconductor solid-state devices is reduced. Combining power from multiple individual amplifier unit cells to obtain the desired power levels is in demand [3]. Such systems typically involve filters for frequency selection and attenuation of other bands [4]. Commonly, the combined signals in the passband are transmitted to the output port, and those in the stopband are reflected into the source. These undesired RFsignal-power reflections could cause additional unwanted intermodulation products through the remixing of such RF power reflections with the local oscillator or power amplifiers [5]. Hence, it is required to absorb the reflection power of the input signals in the stopband.

For the conventional filters, an additional branch is used to reduce the reflection in the stopband [6-8], but these structures cannot be directly applied to the filtering power combiner. Based on the Wilkinson power divider, absorptive branch [5] and parallel-LC resonators [9] are added to realize the reflectionless filtering power dividers. The heat-sinking capacity of these Wilkinson power divider-based structures can be further improved.

With the advantage of suppressing environmental noise and neutralizing electromagnetic interference, balanced components such as balanced filters [10-12], power divider [13-15], and coupler [16], have drawn more and more attention. However, a balun is needed to convert signals between unbalanced and balanced circuits in one system, which occupies an additional area. To solve this problem, circuits with both unbalanced and balanced ports are proposed, such as the unbalanced-to-balanced (UTB) filter [17], [18], diplexer [19], and balanced-to-unbalanced (BTU) power divider [20], these circuits also have the function of common-mode suppression (CMS).

Due to the good isolation between the two output ports, a Gysel power divider can work as a high-power combiner. Based on the Gysel power divider, branch lines with open- and short-circuited stubs are used to realize the filtering function of a BTU power divider [21], but the performance of the CMS and the isolation between the two unbalanced ports needs to be further improved. A BTU power divider is present in [22], whose filtering characteristics are realized by using coupled lines. Nevertheless, the 15-dB CMS bandwidth is narrower than the differential-mode operation bandwidth. Dual-mode ring resonators are used to replace the $\lambda / 4$ transmission lines to obtain high selectivity filtering function in [23]. However, the good CMS and isolation bandwidth should be broadened.

In this paper, a high-selectivity reflectionless UTB filtering power combiner is proposed. It has two transmission zeros near the passband edges leading to high selectivity filtering characteristics. 


\section{Theory Analysis and Design}

The schematic of the proposed high-selectivity reflectionless UTB filtering power combiner is depicted in Fig. 1. The input ports 1 and 4 are two unbalanced ports, the balanced output port $A$ comprises of ports 2 and 3 terminated in $Z_{0}$.

The mixed-mode scattering parameter $S_{\mathrm{mm}}$ for the proposed UTB power combiner is expected to satisfy the matching condition at both balanced and unbalanced ports, good isolation between the unbalanced input ports, good CMS, and out-of-phase equal power combination. To meet the requirements, $\left[S_{\mathrm{mm}}\right]$ can be given as follows according to [24]

$$
\begin{aligned}
{\left[S_{\mathrm{mm}}\right] } & =\left[\begin{array}{llll}
S_{\mathrm{ddAA}} & S_{\mathrm{dsA} 1} & S_{\mathrm{dsA} 4} & S_{\mathrm{dcAA}} \\
S_{\mathrm{sd} 1 \mathrm{~A}} & S_{\mathrm{ss} 11} & S_{\mathrm{ss} 14} & S_{\mathrm{sc} 1 \mathrm{~A}} \\
S_{\mathrm{sd} 4 \mathrm{~A}} & S_{\mathrm{ss} 41} & S_{\mathrm{ss} 44} & S_{\mathrm{sc} 4 \mathrm{~A}} \\
S_{\mathrm{cdAA}} & S_{\mathrm{csA} 1} & S_{\mathrm{csA} 4} & S_{\mathrm{ccAA}}
\end{array}\right] \\
& =\left[\begin{array}{cccc}
0 & \frac{\mathrm{e}^{\mathrm{j}\left(\varphi_{1}+\varphi_{2}\right)}}{\sqrt{2}} & \frac{\mathrm{e}^{\mathrm{j}\left(\varphi_{1}+\varphi_{2}+180^{\circ}\right)}}{\sqrt{2}} & 0 \\
\frac{\mathrm{e}^{\mathrm{j}\left(\varphi_{1}+\varphi_{2}\right)}}{\sqrt{2}} & 0 & 0 & 0 \\
\frac{\mathrm{e}^{\mathrm{j}\left(\varphi_{1}+\varphi_{2}+180^{\circ}\right)}}{\sqrt{2}} & 0 & 0 & 0 \\
0 & 0 & 0 & -1
\end{array}\right],
\end{aligned}
$$

where

$$
\begin{gathered}
S_{\mathrm{ddAA}}=\frac{1}{2}\left(S_{22}+S_{33}-S_{23}-S_{32}\right), \\
S_{\mathrm{dsA} 1}=\frac{\sqrt{2}}{2}\left(S_{21}-S_{31}\right), \\
S_{\mathrm{dsA} 4}=\frac{\sqrt{2}}{2}\left(S_{24}-S_{34}\right),
\end{gathered}
$$

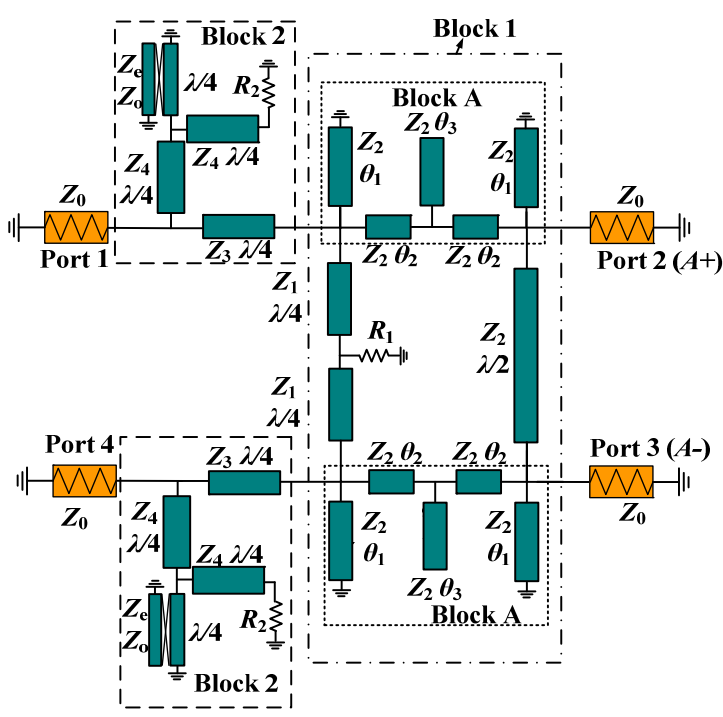

Fig. 1. Schematic of the proposed high-selectivity reflectionless UTB filtering power combiner.

$$
\begin{gathered}
S_{\mathrm{ccAA}}=\frac{1}{2}\left(S_{22}+S_{33}+S_{23}+S_{32}\right), \\
S_{\mathrm{csA} 1}=\frac{\sqrt{2}}{2}\left(S_{21}+S_{31}\right), \\
S_{\mathrm{csA} 4}=\frac{\sqrt{2}}{2}\left(S_{24}+S_{34}\right), \\
S_{\mathrm{cdAA}}=\frac{1}{2}\left(S_{22}-S_{33}-S_{23}+S_{32}\right) .
\end{gathered}
$$

$\varphi_{1}$ and $\varphi_{2}$ are the phase shifts of Block A and Block 2, respectively.

The desired standard scattering matrix $\left[S_{\text {std }}\right]$ of Block 1 can be derived as follows by using (1)-(8),

$$
\begin{aligned}
{\left[S_{\text {std }}\right] } & =\left[\begin{array}{llll}
S_{11} & S_{12} & S_{13} & S_{14} \\
S_{21} & S_{22} & S_{23} & S_{24} \\
S_{31} & S_{32} & S_{33} & S_{34} \\
S_{41} & S_{42} & S_{43} & S_{44}
\end{array}\right] \\
& =\left[\begin{array}{cccc}
0 & \frac{\mathrm{e}^{\mathrm{j} \varphi_{1}}}{2} & \frac{\mathrm{e}^{\mathrm{j}\left(\varphi_{1}+180^{\circ}\right)}}{2} & 0 \\
\frac{\mathrm{e}^{\mathrm{j}\left(\varphi_{1}+180^{\circ}\right)}}{2} & -\frac{1}{2} & -\frac{1}{2} & \frac{\mathrm{e}^{\mathrm{j}\left(\varphi_{1}+180^{\circ}\right)}}{2} \\
0 & \frac{\mathrm{e}^{\mathrm{j}\left(\varphi_{1}+180^{\circ}\right)}}{2} & \frac{\mathrm{e}^{\mathrm{j} \varphi_{1}}}{2} & \frac{\mathrm{e}^{\mathrm{j} \varphi_{1}}}{2} \\
0
\end{array}\right] .
\end{aligned}
$$

\subsection{Theory Analysis of Block 1}

As shown with dashed-dotted-dashed lines in Fig. 1, Block 1 is composed of two filtering sections (i.e., Block A), two transmission lines $\left(Z_{1}, \lambda / 4\right)$, a grounded resistor $\left(R_{1}\right)$, and a phase inverter $\left(Z_{2}, \lambda / 2\right)$. The filtering section consists of two short-circuited stubs $\left(Z_{2}, \theta_{1}\right)$, two transmission lines $\left(Z_{2}, \theta_{2}\right)$, and an open-circuited stub $\left(Z_{2}\right.$, $\left.\theta_{3}\right)$. The simplified circuits in terms of odd- and even-mode of Block 1 are given in Fig. 2.

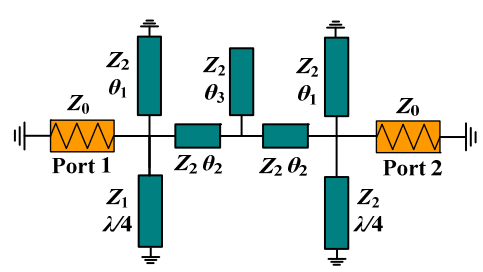

(a)

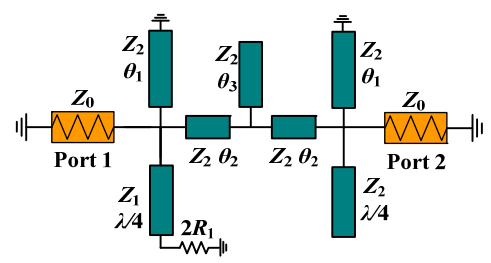

(b)

Fig. 2. Equivalent circuits of Block 1. (a) Odd-mode. (b) Even-mode. 


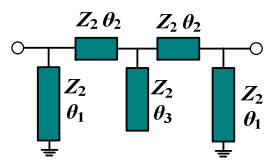

(a)

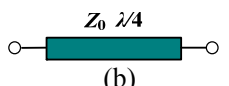

(b)
Fig. 3. (a) Filtering section. (b) $\lambda / 4$ transmission line.

By decomposing the desired standard scattering matrix (9), the scattering matrixes of the odd- and evenmode equivalent circuits of Block 1 are needed as

$$
\begin{gathered}
{\left[S_{\mathrm{o}}\right]=\left[\begin{array}{cc}
0 & 2 S_{21} \\
2 S_{21} & 0
\end{array}\right]=\left[\begin{array}{cc}
0 & \mathrm{e}^{\mathrm{j} \varphi_{1}} \\
\mathrm{e}^{\mathrm{j} \varphi_{1}} & 0
\end{array}\right],} \\
{\left[S_{\mathrm{e}}\right]=\left[\begin{array}{cc}
0 & 0 \\
0 & 2 S_{22}
\end{array}\right]=\left[\begin{array}{cc}
0 & 0 \\
0 & -1
\end{array}\right] .}
\end{gathered}
$$

At the center frequency $f_{0}$, the effects of the two $\lambda / 4$ short-circuited stubs in Fig. 2(a) can be omitted, hence the odd-mode circuit is simplified to be a filtering section in Fig. 3(a), and it can be equivalent to one section of $\lambda / 4$ transmission line as shown in Fig. 3(b) for satisfying the needed $S$-parameters as (10). Based on this equivalent relationship and $A B C D$ matrix analysis, the electrical parameters of the filtering section can be derived.

The $A B C D$ matrix of the filtering section can be derived as

$$
\begin{aligned}
{\left[\begin{array}{ll}
A & B \\
C & D
\end{array}\right]_{\mathrm{a}} } & =\left[\begin{array}{cc}
1 & 0 \\
\frac{1}{\mathrm{j} Z_{2} \tan \theta_{1}} & 1
\end{array}\right]\left[\begin{array}{cc}
\cos \theta_{2} & \mathrm{j} Z_{2} \sin \theta_{2} \\
\mathrm{j} \sin \theta_{2} / Z_{2} & \cos \theta_{2}
\end{array}\right] \\
& \times\left[\begin{array}{cc}
1 & 0 \\
\frac{-1}{\mathrm{j} Z_{2} \cot \theta_{3}} & 1
\end{array}\right]\left[\begin{array}{cc}
\cos \theta_{2} & \mathrm{j} Z_{2} \sin \theta_{2} \\
\mathrm{j} \sin \theta_{2} / Z_{2} & \cos \theta_{2}
\end{array}\right] \\
& \times\left[\begin{array}{cc}
1 & 0 \\
\frac{1}{\mathrm{j} Z_{2} \tan \theta_{1}} & 1
\end{array}\right] .
\end{aligned}
$$

The $A B C D$ matrix of the $\lambda / 4$ transmission line with the characteristic impedance $Z_{0}$ is expressed as

$$
\left[\begin{array}{cc}
A & B \\
C & D
\end{array}\right]_{\mathrm{b}}=\left[\begin{array}{cc}
0 & \mathrm{j} Z_{0} \\
\frac{\mathrm{j}}{Z_{0}} & 0
\end{array}\right] .
$$

For simplicity, $Z_{2}$ is set to be equal to $Z_{0}$.

$$
Z_{2}=Z_{0} \text {. }
$$

By applying the $A B C D$ parameters $A_{\mathrm{a}}=A_{\mathrm{b}}, B_{\mathrm{a}} / C_{\mathrm{a}}=$ $B_{\mathrm{b}} / C_{\mathrm{b}}$, the conditions for the filtering section at $f_{0}$ equivalent to the $\lambda / 4$ transmission line are found as

$$
\begin{aligned}
& \cot \left(2 \theta_{2}\right)+2 \sin \left(2 \theta_{2}\right) \cot \theta_{3}-\sin \left(2 \theta_{2}\right) \tan \theta_{1} \\
& +2 \cos \left(2 \theta_{2}\right) \cot \left(\theta_{3}\right) \tan \theta_{1}-1=0, \\
& 1-4 \cos \left(2 \theta_{2}\right) \cot \theta_{3} \tan \theta_{1}-2 \sin \left(2 \theta_{2}\right) \cot \theta_{3} \\
& -\cos \left(2 \theta_{2}\right)+2 \tan ^{2} \theta_{1}+2 \sin \left(2 \theta_{2}\right) \tan \theta_{1}=0 .
\end{aligned}
$$

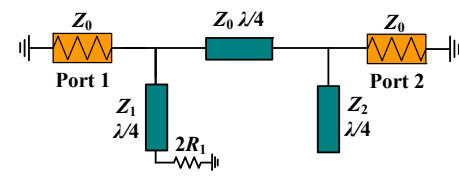

(a)

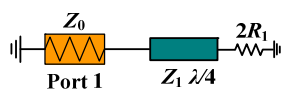

(b)

Fig. 4. (a) Filtering section replaced by $\lambda / 4$ transmission line in the even-mode circuit of Block 1. (b) Simplified circuit.

At $f_{0}$, the filtering section in Fig. 2(b) can be replaced by the $\lambda / 4$ transmission line, as shown in Fig. 4(a). For port 1 , the effects of the $\lambda / 4$ open-circuited stub $\left(Z_{2}\right)$ and the $\lambda / 4$ transmission line $\left(Z_{0}\right)$ in Fig. $4(a)$ at the center frequency can be omitted. Thus, Fig. 4(a) is further simplified as Fig. 4(b).

To meet the matching condition in terms of evenmode $\left(S_{\mathrm{e} 11}=0\right)$, the relation between the characteristic impedance $Z_{1}$ and resistor $R_{1}$ in Fig. 4(b) can be obtained as

$$
Z_{1}=\sqrt{2 R_{1} Z_{0}} \text {. }
$$

To analyze the effect of the electrical lengths on the performance, five cases are given in Tab. 1 calculated with

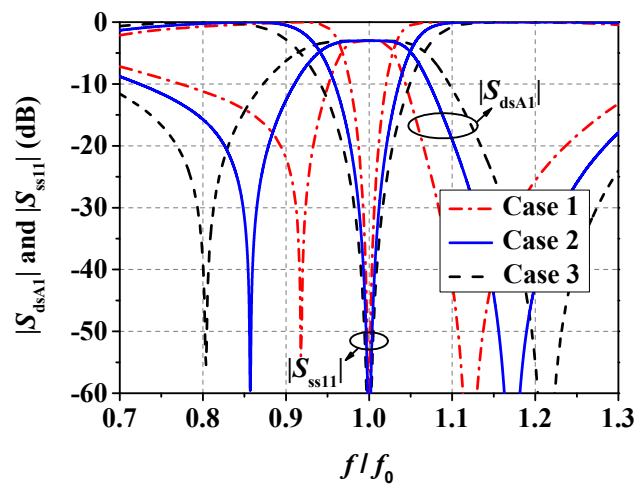

(a)

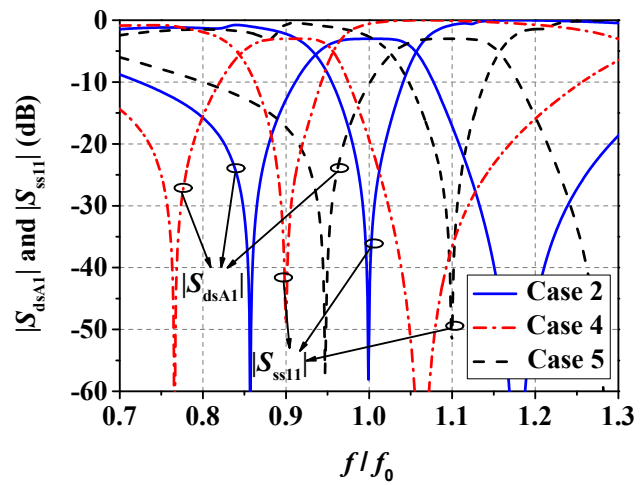

(b)

Fig. 5. Simulated performances of Block 1 with different electrical lengths of the filtering section. (a) Tuning the transmission zeros. (b) Tuning the center frequency of the passband. 


\begin{tabular}{|c|c|c|c|}
\hline Case Number & $\theta_{1}\left({ }^{\circ}\right)$ & $\theta_{2}\left({ }^{\circ}\right)$ & $\theta_{3}\left({ }^{\circ}\right)$ \\
\hline 1 & 159.46 & 15.24 & 98.0 \\
\hline 2 & 152.80 & 18.82 & 105.0 \\
\hline 3 & 147.58 & 21.21 & 112.0 \\
\hline 4 & 169.34 & 21.25 & 117.5 \\
\hline 5 & 139.33 & 16.82 & 95.0 \\
\hline
\end{tabular}

Tab. 1. Electrical lengths of the filtering section with $Z_{2}=Z_{0}$.

(14)-(17). As shown in Fig. 5(a), when $\theta_{3}$ decreases from $112^{\circ}$ to $98^{\circ}$, the transmission zeros of $\left|S_{\mathrm{dsAl}}\right|$ become closer as a result of higher selectivity, but the transmission bandwidth is decreased. Therefore, there is a trade-off between selectivity and bandwidth. Furthermore, the center frequency of the UTB combiner can be flexibly controlled by the electrical lengths $\theta_{1}, \theta_{2}$, and $\theta_{3}$, as shown in Fig. 5(b).

\subsection{Analysis of Block 2}

To achieve the input-port reflectionless response, two absorbing branches (i.e., Block 2, as shown with dash line in Fig. 1) are separately connected at two unbalanced ports (i.e., ports 1 and 4). Each absorbing branch consists of a transmission-line section $\left(Z_{3}, \lambda / 4\right)$, two transmission-line branches $\left(Z_{4}, \lambda / 4\right)$, an absorbing resistor $\left(R_{2}\right)$, and shortcircuited coupled lines $\left(Z_{\mathrm{e}}, Z_{\mathrm{o}}\right.$, and $\left.\lambda / 4\right)$. Owe to the absorbing branch, two additional reflection zeros are generated, which enhance the matching bandwidth of $\left|S_{\text {ddAA }}\right|$, as shown in Fig. 6. Furthermore, the introduction of the absorbing branches will not affect the location of the two transmission zeros.

Figure 7 gives the simulated responses of the proposed reflectionless UTB filtering power combiner with different values of $Z_{4}$. As shown in Fig. 7(a), $Z_{4}$ has effects on the $-10-\mathrm{dB}$ input-reflection absorptive bandwidth (i.e., the bandwidth for $\left|S_{\mathrm{ss} 11}\right|<-10 \mathrm{~dB}$ ), and the input-reflection responses around $0.95 f_{0}$ and $1.04 f_{0}$ becomes poor when $Z_{4}$ decreases from 80 to $40 \Omega$. It can be seen from Fig. 7(b) that a smaller value of $Z_{4}$ causes poor responses of $\left|S_{\mathrm{ddAA}}\right|$ and $\left|S_{\mathrm{dsA} 1}\right|$ in the passband.

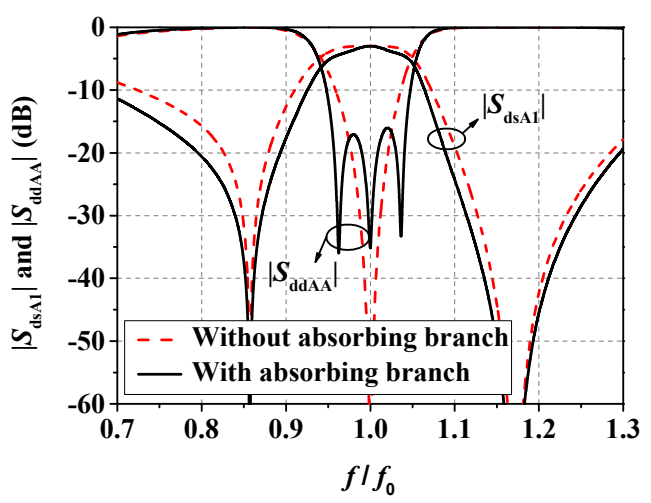

Fig. 6. Simulated performances of the proposed UTB filtering power combiner with or without the absorbing branches.

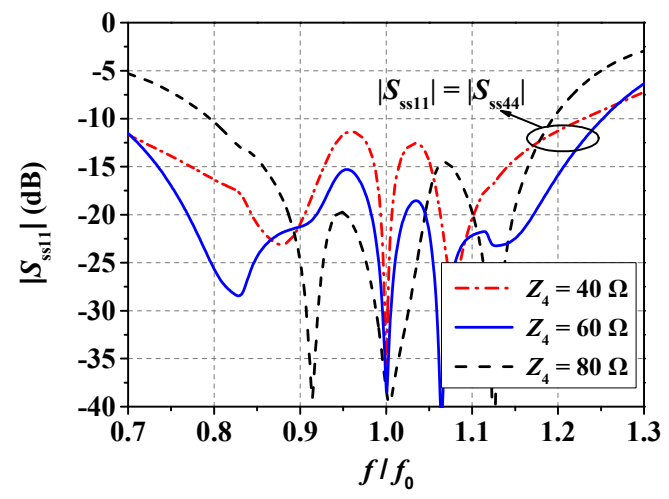

(a)

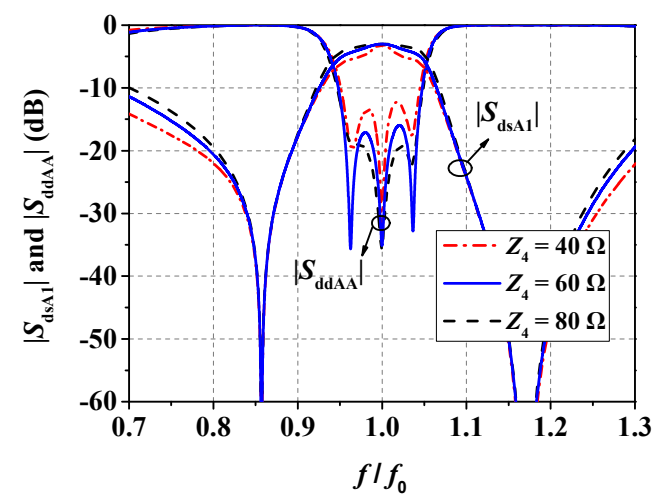

(b)

Fig. 7. Effect of $Z_{4}$ on the performances of the proposed UTB filtering power combiner.

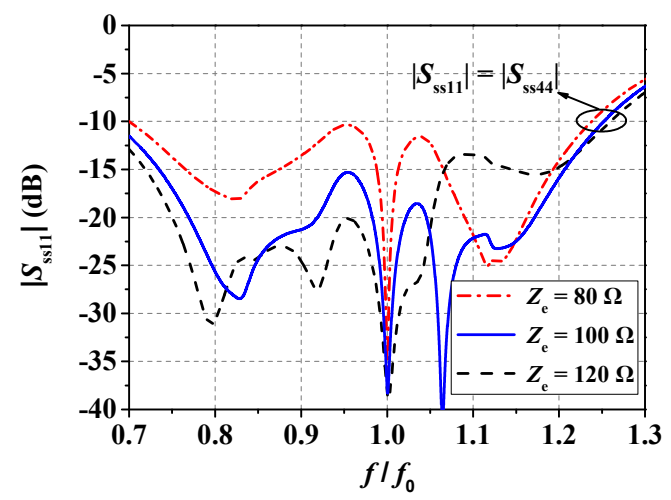

(a)

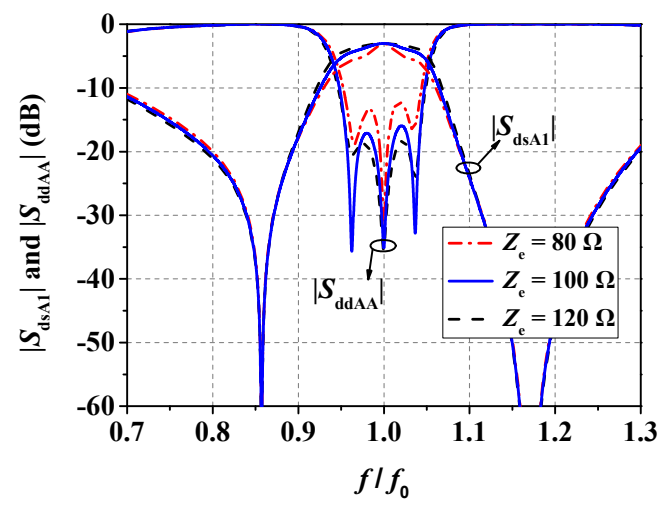

(b)

Fig. 8. Effect of $Z_{\mathrm{e}}$ on the performances of the proposed UTB filtering power combiner. 
Figure 8 gives the simulated responses of the proposed reflectionless UTB filtering power combiner with different values of $Z_{\mathrm{e}}$. As shown in Fig. 8(a), weakly coupling $\left(Z_{\mathrm{e}}=80 \Omega\right.$ and $\left.Z_{\mathrm{o}}=55 \Omega\right)$ affects the input-reflection responses around $0.95 f_{0}$ and $1.04 f_{0}$, and tightly coupling $\left(Z_{\mathrm{e}}=120 \Omega\right.$ and $Z_{\mathrm{o}}=55 \Omega$ ) degrades input-reflection response around $1.09 f_{0}$. It can be found from Fig. 8(b) that $Z_{\mathrm{e}}$ also has effects on the differential-mode return loss and transmission coefficient $\left(\left|S_{\mathrm{ddAA}}\right|\right.$ and $\left.\left|S_{\mathrm{dsA} 1}\right|\right)$ in the passband.

Based on the previous analysis, the following steps are suggested to determine the parameters in Block 2.

1) Choose $Z_{3}=50 \Omega$ to connect ports 1 and $4\left(Z_{0}=\right.$ $50 \Omega$ ) for obtaining ideal impedance matching characteristics at $f_{0}$.

2) Tune $Z_{4}$ to obtain a wide -10 -dB input-reflection absorptive bandwidth, referring to Fig. 7.

3) Select proper $Z_{\mathrm{e}}$ and $Z_{\mathrm{o}}$ to avoid the degraded input-reflection responses in the absorptive bandwidth, referring to Fig. 8.

Finally, the circuit parameters are determined as $Z_{0}=$ $Z_{2}=Z_{3}=50 \Omega, Z_{1}=70.7 \Omega, Z_{4}=60 \Omega, Z_{\mathrm{e}}=100 \Omega, Z_{\mathrm{o}}=$ $55 \Omega, R_{1}=R_{2}=50 \Omega, \theta_{1}=153.6^{\circ}, \theta_{2}=18.79^{\circ}$, and $\theta_{3}=$ $105^{\circ}$.

\section{Implementation and Performance}

To demonstrate the theory in Sec. 2, a microstrip high-selectivity reflectionless UTB filtering power combiner is implemented on a microwave substrate (relative permittivity $\varepsilon_{\mathrm{r}}=2.65$, thickness $h=1.5 \mathrm{~mm}$ ) as shown in Fig. 9. The values of the isolation resistor and absorbing resistor are $R_{1}=R_{2}=50 \Omega$. The center frequency is $f_{0}=$ $1.0 \mathrm{GHz}$. The dimensions are finally obtained with the HFSS electromagnetic simulation, as given in Tab. 2.

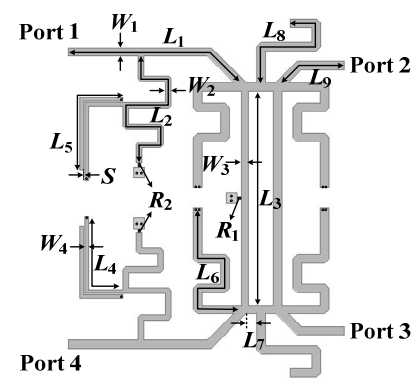

(a)

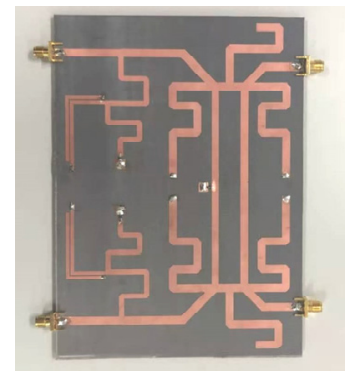

(b)
Fig. 9. Layout and photograph of the proposed highselectivity reflectionless UTB filtering power combiner. (a) Layout. (b) Photograph.

\begin{tabular}{|c|c|c|c|c|c|c|}
\hline$W_{1}$ & $L_{1}$ & $W_{2}$ & $L_{2}$ & $W_{3}$ & $L_{3}$ & $W_{4}$ \\
\hline 4.1 & 86.5 & 2.7 & 109.9 & 3.5 & 101.6 & 1.79 \\
\hline$L_{4}$ & $L_{5}$ & $L_{6}$ & $L_{7}$ & $L_{8}$ & $L_{9}$ & $S$ \\
\hline 51.2 & 54.5 & 94.5 & 4.95 & 66.0 & 32.5 & 0.56 \\
\hline
\end{tabular}

Tab. 2. Dimensions of the proposed high-selectivity reflectionless UTB filtering power combiner (unit: $\mathrm{mm}$ ).

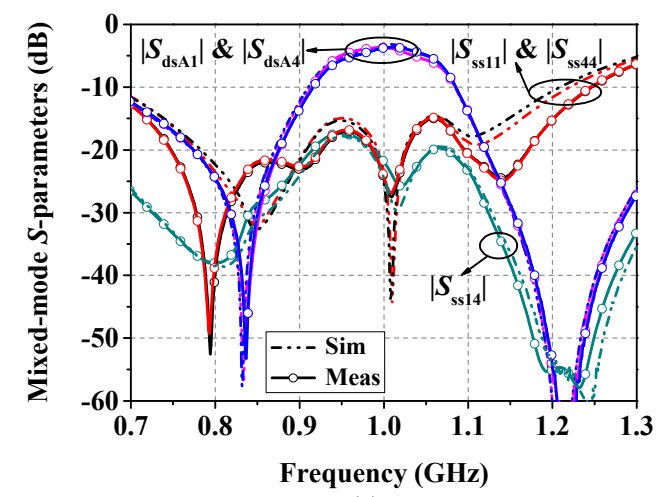

(a)

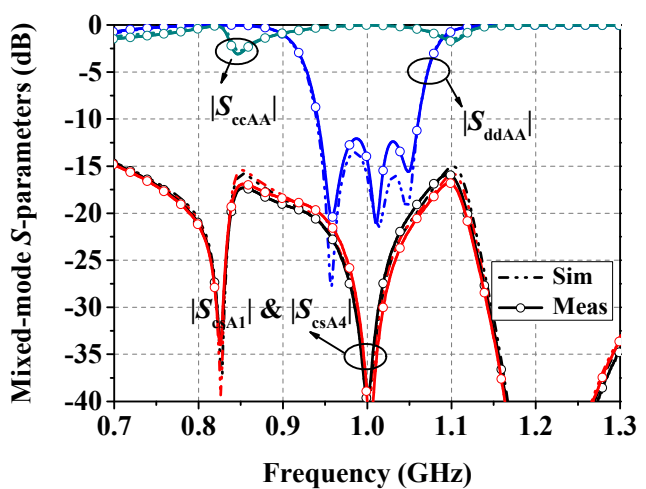

(b)

Fig. 10. Simulation and measurement curves of the proposed UTB filtering power combiner. (a) Unbalanced-port responses. (b) Balanced-port responses.

Simulation and measurement curves of the proposed UTB filtering power combiner are given in Fig. 10. For the unbalanced-port responses, the measured 3-dB transmission band of $\left|S_{\mathrm{dsA} 1}\right|$ and $\left|S_{\mathrm{dsA} 4}\right|$ is $933-1065 \mathrm{MHz}$ (thirteen-point-two percent). The $-10-\mathrm{dB}$ absorptive band of input-reflection is $700-1245 \mathrm{MHz}$ (fifty-five percent), which is 4.12 times the transmission band. The isolation is $24.6 \mathrm{~dB}$ at $f_{0}$, and better than $17.8 \mathrm{~dB}$ from 0.7 to $1.3 \mathrm{GHz}$. Two transmission zeros at 0.838 and $1.215 \mathrm{GHz}$ are obtained. The attenuation rates (AR) are 137.1 and $140.5 \mathrm{~dB}$, respectively, where the AR is defined as

$$
\mathrm{AR}=\frac{20 \mathrm{~dB}-3 \mathrm{~dB}}{\left|f_{20 \mathrm{~dB}}-f_{3 \mathrm{~dB}}\right| / f_{0}} .
$$

For the balanced-port responses, the CMS is better than $15 \mathrm{~dB}$ from 0.7 to $1.3 \mathrm{GHz}$, with excellent CMS of $40.1 \mathrm{~dB}$ at $f_{0}$. The 10-dB return-loss band of the differential mode is $941-1061 \mathrm{MHz}$ (twelve percent). Figure 11 gives the simulation and measurement curves of the phase difference between $S_{\mathrm{dsA} 4}$ and $S_{\mathrm{dsA} 1}$. The measured phase difference between the unbalanced ports is $180^{\circ} \pm 0.4^{\circ}$ from 884 to $1100 \mathrm{MHz}$.

The comparisons among AR, CMS, isolation, 3-dB transmission bandwidth, 15-dB CMS bandwidth, circuit size, and whether with reflectionless response are summarized in Tab. 3. Compared with the previous works, the proposed UTB filtering power combiner has sharp edges at 


\begin{tabular}{|c|c|c|c|c|c|c|c|c|c|}
\hline Refs. & Type & $\begin{array}{c}f_{0} \\
(\mathrm{GHz})\end{array}$ & $\begin{array}{c}\mathrm{AR}_{\text {low }} / \mathrm{AR}_{\text {up }} \\
(\mathrm{dB})\end{array}$ & $\begin{array}{c}\mathrm{CMS} \\
(\mathrm{dB})\end{array}$ & $\begin{array}{c}\text { Isolation } \\
(\mathrm{dB})\end{array}$ & $\begin{array}{c}3-\mathrm{dB} \text { FBW } \\
(\%)\end{array}$ & $\begin{array}{c}15-\mathrm{dB} \text { CMS } \\
\mathrm{FBW}(\%)\end{array}$ & $\begin{array}{c}\text { Circuit Size } \\
\left(\lambda_{\mathrm{g}} \times \lambda_{\mathrm{g}}\right)\end{array}$ & $\begin{array}{c}\text { Reflectionless } \\
\text { Response }\end{array}$ \\
\hline$[5]$ & UTU & 2.0 & $85 / 90$ & - & 20 & 8 & - & $1.59 \times 1.59$ & Yes \\
\hline$[9]$ & UTU & 1.0 & $71 / 113$ & - & 18 & 15 & - & $0.62 \times 0.75$ & Yes \\
\hline$[21]$ & UTB & 1.88 & $50 / 168$ & 24 & 18 & 6.3 & 7 & $0.70 \times 0.30$ & No \\
\hline$[22]$ & UTB & 2.0 & $112 / 114$ & 35 & 25 & 82 & 25 & $0.52 \times 0.26$ & No \\
\hline$[23]$ & UTB & 3.5 & $114 / 220$ & 55 & 40 & 12 & 7 & $1.24 \times 0.76$ & No \\
\hline $\begin{array}{c}\text { This } \\
\text { work }\end{array}$ & UTB & 1.0 & $137.1 / 140.5$ & 40.1 & 24.6 & 13.2 & 60 & $0.63 \times 0.88$ & Yes \\
\hline
\end{tabular}

Tab. 3. Performance comparisons of the proposed circuit with previous works.

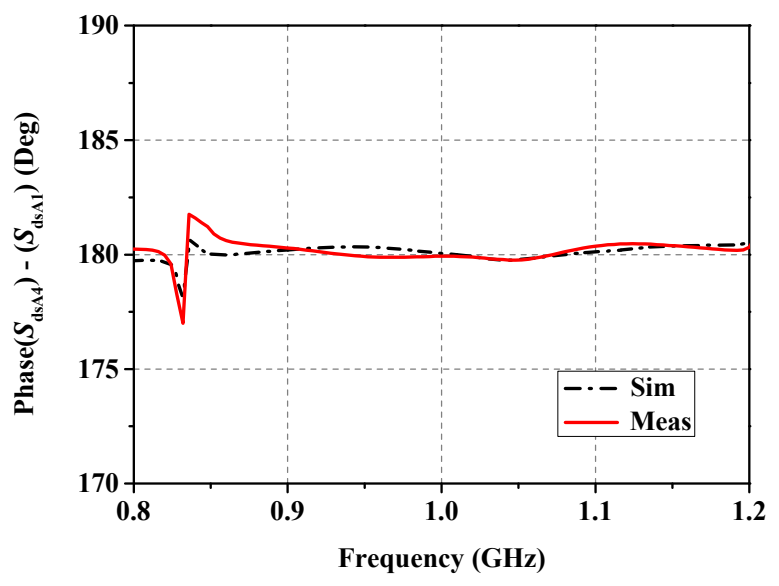

Fig. 11. Phase difference between $S_{\mathrm{dsA} 4}$ and $S_{\mathrm{dsA} 1}$.

both lower and upper sides of the passband and wide CMS bandwidth. A reflectionless response is obtained to avoid the input-reflection.

\section{Conclusion}

In this paper, a high-selectivity reflectionless UTB filtering power combiner has been presented. The designed circuit can achieve the following advantages: (i) an inputreflection absorptive bandwidth is 4.12-times 3-dB transmission bandwidth, (ii) high-selectivity filtering characteristics, (iii) excellent common-mode suppression and isolation within the whole input-reflection absorptive band. In addition, the proposed power combiner also can work as a high-selectivity filtering power divider from the balanced port to the unbalanced ports. For high power applications, the grounded resistors of the proposed power combiner can be external to the packaging structure for efficient heat dissipation [25] or implemented with microwave highpower terminations [26].

\section{Acknowledgments}

This work was supported by National Natural Science Foundation of China (61871417 and 51809030), LiaoNing
Revitalization Talents Program (XLYC2007024 and XLYC2007067), Natural Science Foundation of Liaoning Province (2019-MS-024 and 2020-MS-127), and Fundamental Research Funds for the Central Universities (3132021234 and 3132021231).

\section{References}

[1] TSENG, C., CHANG, C. Improvement of return loss bandwidth of balanced amplifier using metamaterial-based quadrature power splitters. IEEE Microwave and Wireless Components Letters, 2008, vol. 18, no. 4, p. 269-271. DOI: 10.1109/LMWC.2008.918914

[2] GAO, S., GARDNER, P. Integrated antenna/power combiner for LINC radio transmitters. IEEE Transactions on Microwave Theory and Techniques, 2005, vol. 53, no. 3, p. 1083-1088. DOI: 10.1109/TMTT.2005.843491

[3] XU, J., CUI, Y., QIAN, C., et al. A Ka-band power-combined amplifier based on a six-way quasi-planar power divider/combiner. In Asia-Pacific Microwave Conference. Nanjing, (China), 2015, p. 1-3. DOI: 10.1109/APMC.2015.7411821

[4] ROSENBERG, U., SALEHI, M., BORNEMANN, J., et al. A novel frequency-selective power combiner/divider in singlelayer substrate integrated waveguide technology. IEEE Microwave Wireless Components Letters, 2013, vol. 23, no. 8, p. 406-408. DOI: 10.1109/LMWC.2013.2269039

[5] GÓMEZ-GARCÍA, R. MUÑOZ-FERRERAS, J., PSYCHOGIOU, D. RF reflectionless filtering power dividers. IEEE Transactions on Circuits and Systems II: Express Briefs, 2019, vol. 66, no. 6, p. 933-937. DOI: 10.1109/TCSII.2018.2875172

[6] KHALAJ-AMIRHOSSEINI, M., TASKHIRI, M.-M. Twofold reflectionless filters of inverse-Chebyshev response with arbitrary attenuation. IEEE Transactions on Microwave Theory and Techniques, 2017, vol. 65, no. 11, p. 4616-4620. DOI: 10.1109/TMTT.2017.2716940

[7] PSYCHOGIOU, D., GÓMEZ-GARCÍA, R. Reflectionless adaptive RF filters: Bandpass, bandstop, and cascade designs. IEEE Transactions on Microwave Theory and Techniques, 2017, vol. 65 , no. 11, p. 4593-4605. DOI: 10.1109/TMTT.2017.2734086

[8] PSYCHOGIOU, D., GÓMEZ-GARCÍA, R. Tunable reflectionless microstrip bandpass filters. In IEEE Radio and Wireless Symposium (RWS). Anaheim (USA), 2018, p. 49-51. DOI: 10.1109/RWS.2018.8304943 
[9] LEE, B., NAM, S., LEE, J. Filtering power divider with reflectionless response and wide isolation at output ports. IEEE Transactions on Microwave Theory and Techniques, 2019, vol. 67, no. 7, p. 2684-2692. DOI: 10.1109/TMTT.2019.2913650

[10] ZHANG, W., WU, Y., LIU, Y., et al. Planar wideband differentialmode bandpass filter with common-mode noise absorption. IEEE Microwave and Wireless Components Letters, 2017, vol. 27, no. 5, p. 458-460. DOI: 10.1109/LMWC.2017.2690839

[11] FENG, W., MA, X., CHE, W., et al. Narrow-band balanced filtering network using coupled lines loaded with stubs. Electronics Letters, 2018, vol. 54, no. 6, p. 366-368. DOI: 10.1049/el.2017.4628

[12] GAO, X., FENG, W., CHE, W. High-selectivity wideband balanced filters using coupled lines with open/shorted stubs. IEEE Microwave and Wireless Components Letters, 2017, vol. 27, no. 3, p. 260-262. DOI: 10.1109/LMWC.2017.2661998

[13] XIA, B., WU, L., MAO, J. A new balanced-to-balanced power divider/combiner. IEEE Transactions on Microwave Theory and Techniques, 2012, vol. 60, no. 9, p. 2791-2798. DOI: 10.1109/TMTT.2012.2203926

[14] XIA, B., WU, L., REN, S., et al. A balanced-to-balanced power divider with arbitrary power division. IEEE Transactions on Microwave Theory and Techniques, 2013, vol. 61, no. 8, p. 2831-2840. DOI: 10.1109/TMTT.2013.2268739

[15] LI, M., WU, Y., JIAO, L., et al. A planar balanced-to-balanced power divider with wideband filtering responses and commonmode suppressions. IEEE Access, 2018, vol. 6, p. 42057-42065. DOI: $10.1109 /$ ACCESS.2018.2859230

[16] JIAO, L., WU, Y., ZHANG, W., et al. Design methodology for six-port equal/unequal quadrature and rat-race couplers with balanced and unbalanced ports terminated by arbitrary resistances. IEEE Transactions on Microwave Theory and Techniques, 2018, vol. 66, no. 3, p. 1249-1262. DOI: 10.1109/TMTT.2017.2778108

[17] LI, H.-Y., XU, J.-X., ZHANG, X. Y. Single-to-balanced and balanced-to-balanced dual-channel filters using multilayer substrate integrated waveguide cavities. IEEE Transactions on Industrial Electronics, 2021, vol. 68, no. 3, p. 2389-2399. DOI: 10.1109/TIE.2020.2975490

[18] CHI, P., YANG, T. Novel 1.5-1.9 GHz tunable single-to-balanced bandpass filter with constant bandwidth. IEEE Microwave and Wireless Components Letters, 2016, vol. 26, no. 12, p. 972-974. DOI: 10.1109/LMWC.2016.2623251

[19] CHI, P., YANG, T. Novel 1.5-2.4 GHz tunable single-to-balanced diplexer. IEEE Microwave and Wireless Components Letters, 2016, vol. 26, no. 10, p. 783-785. DOI: 10.1109/LMWC.2016.2601279

[20] WU, L., GUO, Y., QIU, L., et al. A new balanced-to-single-ended (BTSE) power divider. In IEEE International Wireless Symposium (IWS). Xi'an (China), 2014, p. 1-4. DOI: 10.1109/IEEEIWS.2014.6864182

[21] XU, K., SHI, J., LIN, L., et al. A balanced-to-unbalanced microstrip power divider with filtering function. IEEE Transactions on Microwave Theory and Techniques, 2015, vol. 63, no. 8, p. 2561-2569. DOI: 10.1109/TMTT.2015.2445051

[22] GAO, X., FENG, W., CHE, W., et al. Wideband balanced-tounbalanced filtering power dividers based on coupled lines. IEEE Transactions on Microwave Theory and Techniques, 2017, vol. 65, no. 1, p. 86-95. DOI: 10.1109/TMTT.2016.2614668

[23] FENG, W., CHE, W., SHI, Y., et al. High selectivity balanced-tounbalanced filtering power dividers using dual-mode ring resonators. IEEE Transactions on Components, Packaging and Manufacturing Technology, 2019, vol. 9, no. 5, p. 927-935. DOI: 10.1109/TCPMT.2018.2866129
[24] EISENSTADT, W. R., STENGEL, B., THOMPSON, B. M. Microwave Differential Circuit Design Using Mixed-Mode SParameters. Boston (USA): Artech House, 2006. ISBN: 9781580539333

[25] ABBOSH, A. M. Planar out-of-phase power divider/combiner for wideband high power microwave applications. IEEE Transactions on Components, Packaging and Manufacturing Technology, 2014, vol. 4, no. 3, p. 465-471. DOI: 10.1109/TCPMT.2013.2277587

[26] BEIJING BED-TECH MANUFACTURE CO., LTD. Micro RF Terminations (datasheet). [Online]. Available at: http://www.rfbed.com

\section{About the Authors ...}

Zihui ZHU was born in Nanping, Fujian, China. He received the B.Eng. degree in Electronic Information Science and Technology from Dalian Maritime University (DMU), Liaoning, China, in 2016. He is currently studying for the Ph.D. degree at DMU. His current research interests focus on power divider/combiner and balanced components.

Zhongbao WANG (corresponding author) received the Ph.D. degree in Communication and Information Systems from DMU in 2012. He is currently an Associate Professor with the School of Information Science and Technology, DMU. From 2014 to 2018, he was a Postdoctoral Fellow with Beijing University of Posts and Telecommunications, China. From 2019 to 2020, he was a Visiting Scholar with National University of Singapore, Singapore. His current research interests include passive microwave components and antennas. He has authored more than 80 journal and conference papers. He is currently serving as a Technical Reviewer for the IEEE Trans. on MTT, AWPL, IET MAP, MOTL, and Radioengineering. He was a recipient of the Best Doctor's Dissertation Award of Liaoning Province in 2013.

Yu BAI was born in Jiaohe, Jilin, China. He received the B.Eng. degree in Electronic Information Engineering from DMU in 2019. He is currently studying for the M.Eng. degree at DMU. His current research interests focus on microwave circuits.

Hongmei LIU received the Ph.D. degree in Information and Communication Engineering from DMU in 2016. She is currently an Associate Professor with the School of Information Science and Technology, DMU. Her current research interests include passive microwave circuits and $\mathrm{CP}$ microwave antennas.

Shaojun FANG received the Ph.D. degree in Communication and Information Systems from DMU in 2001. Since 1982, he has been with DMU, where he is currently the Head Professor with the School of Information Science and Technology. His recent research interests include passive $\mathrm{RF}$ components and patch antennas. He has authored more than 120 papers in journals and conferences. He was a recipient of the Best Doctor's Dissertation Award of Liaoning Province in 2002 and the Outstanding Teacher Award of the Ministry of Transport of China. 